\title{
FDG-PET scan in lymphoma: silver anniversary
}

\author{
Alberto Biggi $^{1} \cdot$ Andrea Gallamini $^{2}$
}

Received: 19 March 2015 / Accepted: 18 June 2015/Published online: 9 July 2015

(C) Italian Association of Nuclear Medicine and Molecular Imaging 2015

Coming together is a beginning. Keeping together is progress. Working together is success.

Henry Ford

Since its first proposal in the late 1980s FDG-PET scan (and later on FDG-PET/CT scan) and lymphoma have become an indissoluble binomial. FDG-PET scan proves a very useful tool for Hodgkin (HL) and non-Hodgkin (NHL) lymphoma staging, restaging and prognostication and is essential for radiotherapy planning and chemosensitivity assessment in HL; the last-born offspring of this synergy has been the PET response-adapted strategy in HL and diffuse large B cell lymphoma (DLBCL). Thanks also to the contribution of PET scan in all these important steps of lymphoma management, the longterm lymphoma survivorship has increased overtime in the last two decades also in lymphoma subsets (elderly patients and HL) in which no major advances in treatment effectiveness could have account for this improvement.

Onco-haematologists and nuclear medicine experts joined their efforts in several informal and international workshops dedicated to PET scan in lymphoma to standardize interim and end-of therapy PET scan reporting, proposing the so-called "Deauville five-point scale" or "Deauville rules", from the venue hosting the first meeting. The latter, in turn, has been incorporated in the lastborn criteria for lymphoma staging and restaging, the so-

Alberto Biggi

biggi.a@ospedale.cuneo.it

1 Nuclear Medicine Department, S. Croce e Carle Hospital, Cuneo, Italy

2 Research, Innovation and Statistics Department, A. Lacassagne Cancer Centre, Nice, France called Lugano criteria, which have definitely included FDG-PET scan in the armamentarium of the necessary imaging tools for assessing the tumour spread in FDG-avid lymphoma. In agreement with the organization committee of the International Conference on Malignant Lymphoma (ICML), which is held every other year in Lugano, the PET in lymphoma workshop is held every other year in Menton (France), interspersed with Lugano meetings. The cooperation between haematologists and nuclear medicine experts has been further tightened in the organization of dedicated courses for PET reporting in clinical trials based on PET imaging.

The technological evolution of PET scanning equipment, the implementation of new software able to perform a semi-automatic reading of FDG concentrating lesions, the new metrics for quantitative PET scan (Q-PET) reading with SUV and derived parameters such as metabolic tumour volume (MTV) and total lesion glycolysis (TLG) have further widened the interdisciplinary cooperation, involving other categories of imaging experts: the medical physicists, the bioinformatics and the statisticians.

The progress of bioinformatics has eased the imaging exchange for central review of scans and central handling and computing of Q-PET parameters. This in turn has opened new frontiers for international cooperation among cooperative groups in lymphoma, in the context of PETbased multicentre clinical trials.

Finally, the new trends in personalized medicine have spurred the search for newer, more accurate tools for prognostication on a single-patient basis, and biomarkers have been recently integrated with imaging for the setup of newer prognostic model. For this reason, the galaxy of the expanding knowledge revolving around imaging has spurred an interdisciplinary approach needing dedicated channels of information. 
The published reports of international committees follow the evolution of the use of PET in lymphoma. In the late 1980s, FDG for PET/CT scan was described as a "suitable radiopharmaceutical for detection of non-Hodgkin's lymphomas that fulfilled the criteria of the ideal tumorophilic radiocompound expensive to produce" [1]. Twenty years later, FDG-PET scan was "strongly recommended before treatment for patients with routinely FDGavid, potentially curable lymphomas to better delineate the extent of disease, .... to predict therapeutic outcome when performed after one to four cycles of multiagent chemotherapy, ... essential for the post-treatment assessment of DLBCL and Hodgkin's lymphoma" [2]. In 2014, FDG-PET was "recommended for routinely staging FDG avid lymphoma in clinical practice and clinical trials, it was deemed ...superior to CT alone to assess early response, ....and standard of care for remission assessment in FDGavid lymphoma" [3].
Conflict of interest Alberto Biggi and Andrea Gallamini have no conflicts of interest.

\section{References}

1. Lister TA, Crowther D, Sutckiffe SB et al (1989) Report of a committee convened to discuss the evaluation and staging of patients with Hodgkin's disease: Cotswolds meeting. J Clin Oncol 7(11):1630-1636

2. Cheson BD, Pfistner B, Juweid ME, Gascoyne RD, Specht L, Horning SJ et al (2007) Revised response criteria for malignant lymphoma. J Clin Oncol 25:579-586

3. Barrington SF, Mikhaeel NG, Kostakoglu L et al (2014) Role of imaging in the staging and response assessment of lymphoma: consensus of the International Conference on Malignant Lymphomas Imaging Working Group. J Clin Oncol 32(27):3048-3058 